\title{
Virtual Endoscopy of Mucin-Producing Pancreas Tumors
}

\author{
Toshio Nakagohri, Ferenc A. Jolesz, Shigeo Okuda, *Takehide Asano, \\ *Takashi Kenmochi , *Osamu Kainuma, *Yoshiharu Tokoro *Hiromichi Aoyama, \\ **William E.Lorensen, Ron Kikinis \\ Department of Radiology Brigham and Women's Hospital, Harvard \\ Medical School \\ * Department of Surgery Chiba University School of Medicine \\ **General Electric Corporate Research and Development Center \\ e-mail:nakagori@med.m.chiba-u.ac.jp
}

\begin{abstract}
We have used computer based virtual endoscopy techniques as a novel approach to clarify the 3D surgical anatomy of the pancreas and improve preoperative surgical planning. 13 cases (18 lesions) with mucinproducing pancreas tumors were investigated by "virtual pancreatoscopy". All cystic tumors and the pancreatic ducts were displayed by virtual endoscopy. The surfaces of intraductal papillary adenocarcinomas were illustrated more irregularly than benign cystic lesions. Virtual pancreatoscopy was useful for surgical planning of minimally invasive resection of the pancreas.
\end{abstract}

\section{Introduction}

Virtual Endoscopy is a new technology which can make computer simulated endoscopic images by processing high resolusion MRI/CT data. Preliminaly study about virtual endoscopy revealed that this technique had many promising advantages. For example, virtual endoscopy can generate views that are not possible in an actual endoscopic examination. Clinical applications of virtual endoscopy for pancreas have not been reported. In this study, usefulness of virtual endoscopy of mucin-producing tumor of the pancreas was studied.

\section{Patients and Methods}

13 cases with mucin-producing pancreas tumor(MPPT) were studied. Two patients have 3 cystic lesions and one patient have 2 lesions, so we investigated 18 lesions with using virtual endoscopy. All patients underwent resection of the pancreas in Chiba University Hospital from 1996 to 1997 . Each patient's profile is in Table 1 
Table 1 Patient's profile

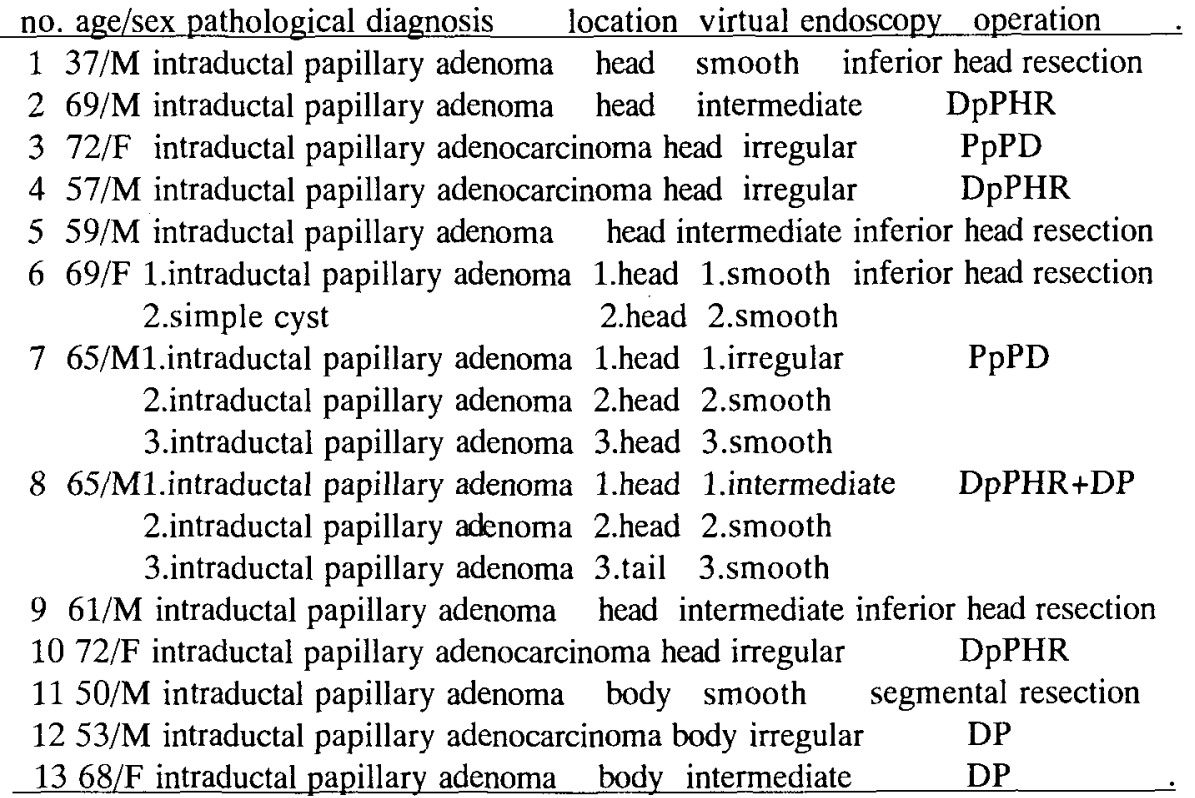

Abbreviation :

DpPHR: duodenum-preserving pancreas head resection

PpPD: pylorus-preserving pancreatoduodenectomy

DP: distal pancreatectomy

The MRI data were acquired by $1.5 T$ Signa MR system(GE). The diagnosis of MPPT was established by findings during endoscopic retrograde cholangio-pancreatography (ERCP) and pathological findings. Virtual endoscopy images were generated with Virtual Endoscopy Software Aplication (VESA) on UNIX workstations in surgical planning laboratory,Department of Radiology and MRI Unit, Brigham and Women's Hospital/Harvard Medical School.

Each image was visualized by processing cross-sectional MRI date according to the threshold of the signal intensity. We used an automated path planning technique to generate path $1,2,3)$.

\section{Patients and Methods}

It was possible to visualize all the pancreatic ducts and cystic lesions of mucin-producing pancreas tumor (MPPT) by virtual endoscopy. The pancreatic ducts and 18 cystic lesions were detected correctly. Virtual endoscopy allowed us to observe the pancreatic duct and the inner surface of the cystic lesions (Fig1,2) . 
Fig. 1: Virtual endoscopy image of the pancreatic duct. A normal pancratic duct was visualized.

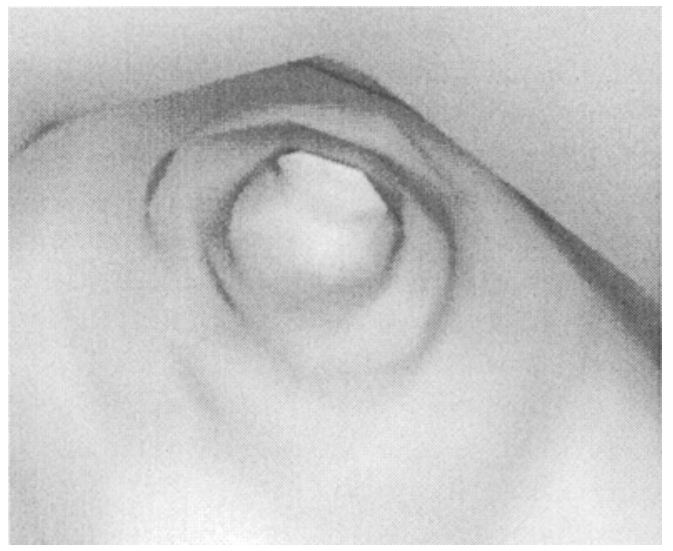

Fig. 2: Virtual endoscopy image of the mucin-producing pancreas tumor. Virtual endoscopy demonstrated the pancreatic duct(right side) and the cystic lesion(left side) simultaneously.

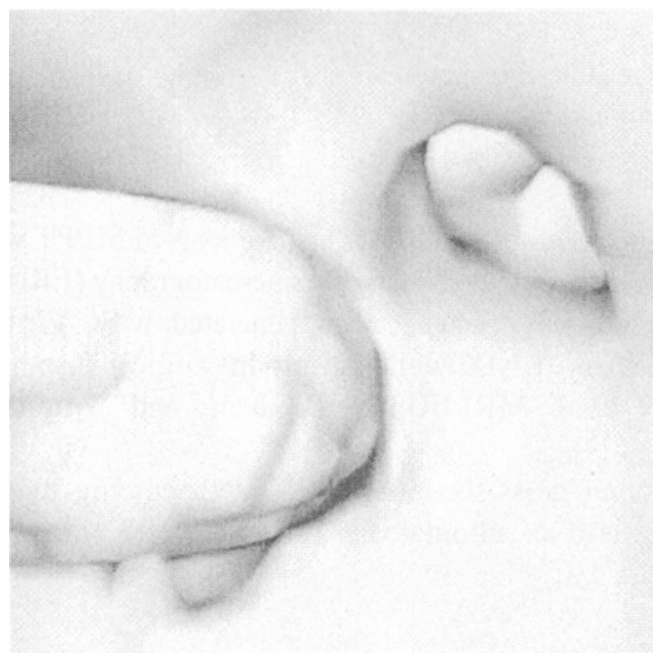

Virtual endoscopy was useful to clarify the anatomical relationship between the cystic tumor ,the pancreatic duct and the bile duct. Eighteen cystic lesions were divided into 3 groups according to the degrees of the irregularity of the surface images rendered by virtual endoscopy: irregular type(n=5)(Fig. 3), intermediate type(n=5)(Fig. 4), and smooth type(n=8)(Fig. 5). 
Fig. 3 : Virtual endoscopy image of irregular type(case 4)

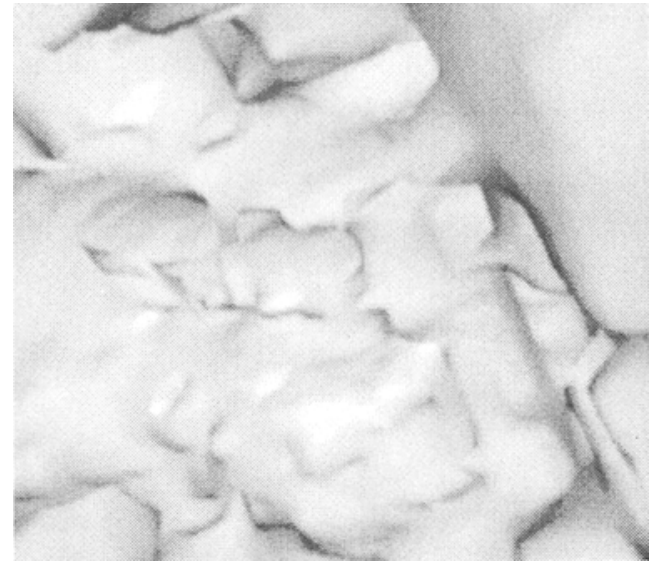

Fig. 4 : Virtual endoscopy image of intermediate type(case 9)

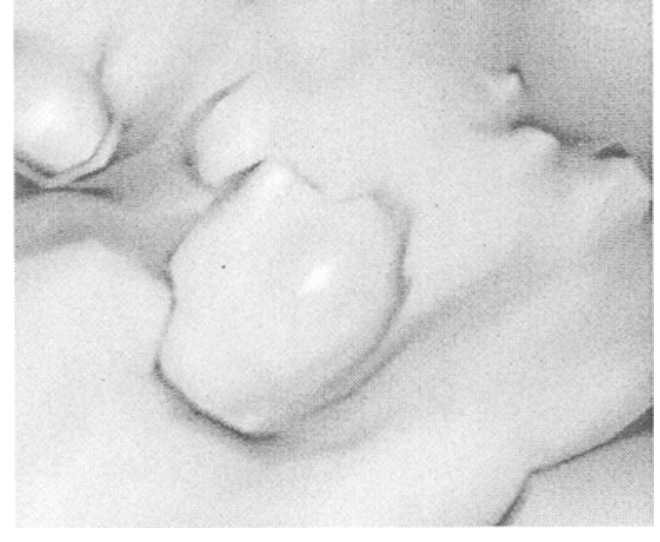

Fig. 5 : Virtual endoscopy image of smooth type(case 11) 
Four lesions of irregular type $(n=5)$ were intraductal papillary adenocarcinomas, and the other irregular lesion was a intraductal papillary adenoma. All five lesions of intermediate type were intraductal papillary adenomas. Six lesions of smooth types were intraductal papillary adenomas, and one lesion of smooth type was a simple cyst. This result revealed that surfaces of adenocarcinomas were illustrated more irregularly than benign cystic lesions by virtual endoscopy. Cystc lesions next to the pancreatic duct were visualized together with the pancreatic duct(Fig.6), because thin septa between cystic lesions and the pancreatic ducts could not be illustrated.

Fig. 6 : A cystic lesion next to the pancreatic duct was generated together with the pancreatic duct.Septum between the panceatic duct and cystic lesion was illustrated like pillars in some cases.
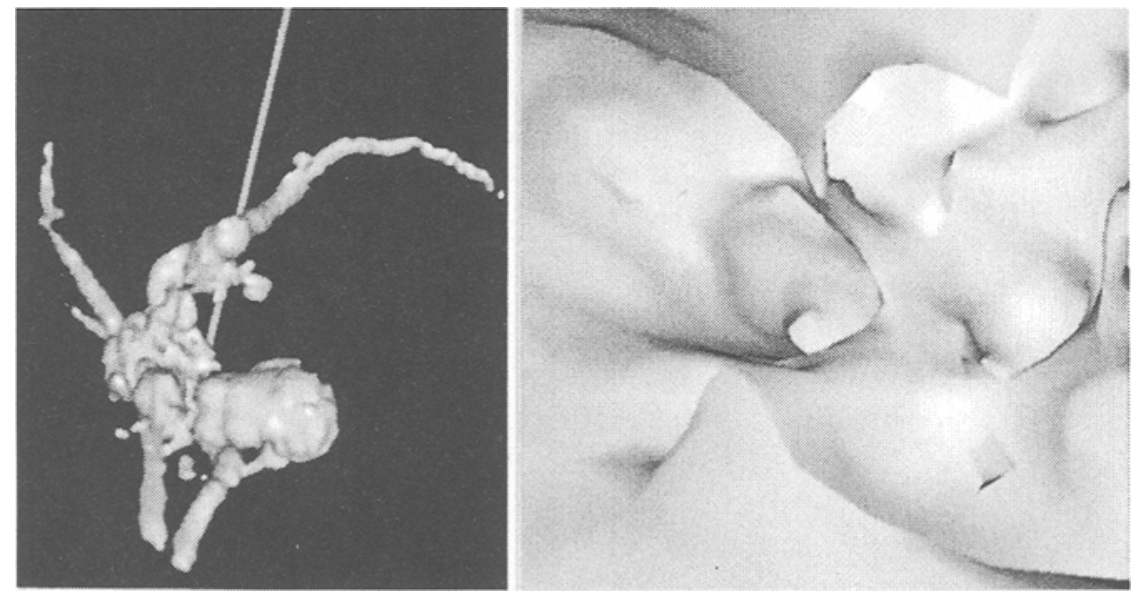

\section{Discussion}

There are two technical approaches for virtual endoscopy: Perspective volume rendering and surface rendering. To improve interpretation and exploration, we have made surface-rendered images derived from computer reconstruction of the cross-sectional MRI image data1,2,3). Walls of fluid-containing organs, such as the pancreatic duct and the bile duct are well demarcated in MRI and CT. Virtual endoscopy depicts the surfaces of the pancreatic duct, the bile duct and the cystic lesions in the pancreas. We can explore the inner space of the pancreatic ducts and cystic lesions interactively. Our virtual endoscopy technique can demonstrate not only a surface rendered image but also a three dimensional (3D) reconstructed image of the pancreas. Therefore, the relationship to anatomic structures located outside the surfaces is continuously maintained and displayed at the same time. Changing the transparency of the images assist the operator to view the bile duct and cystic tumors from the inner space of the pancreatic duct. The viewer may penetrate the walls and see the extent of lesions within and beyond the walls as well as 
the adjacent organs, such as the bile duct and the duodenum. We can also overview the pancreatic duct from the outside at the same time using split screen display (Fig.7).This technique can generate 3D reconstructed images of the pancreatic duct and the bile duct. The operator of virtual endoscopy can perceive his own position. Precise and interactive 3D images also provide information useful for the surgical procedure4,5). Multiwindow display helps surgeons to recognize the anatomical relationship among the cystic lesion, the pancreatic duct and the bile duct. Conventional MRI produce flat, two-dimensional images that give a poor picture of the location and the extent of the tumor in the pancreas.

Fig. 7 : Our virtual endoscopy technique can make 3D reconstructed images of the pancreatic duct and the bile duct. Split screen display helps surgeon to recognize the anatomical relationship among the cystic lesion,the pancreatic duct, and the bile duct. 3-D images and virtual endoscopy images aid diagnosis and surgical planning of mucin-producing pancreas tumors.
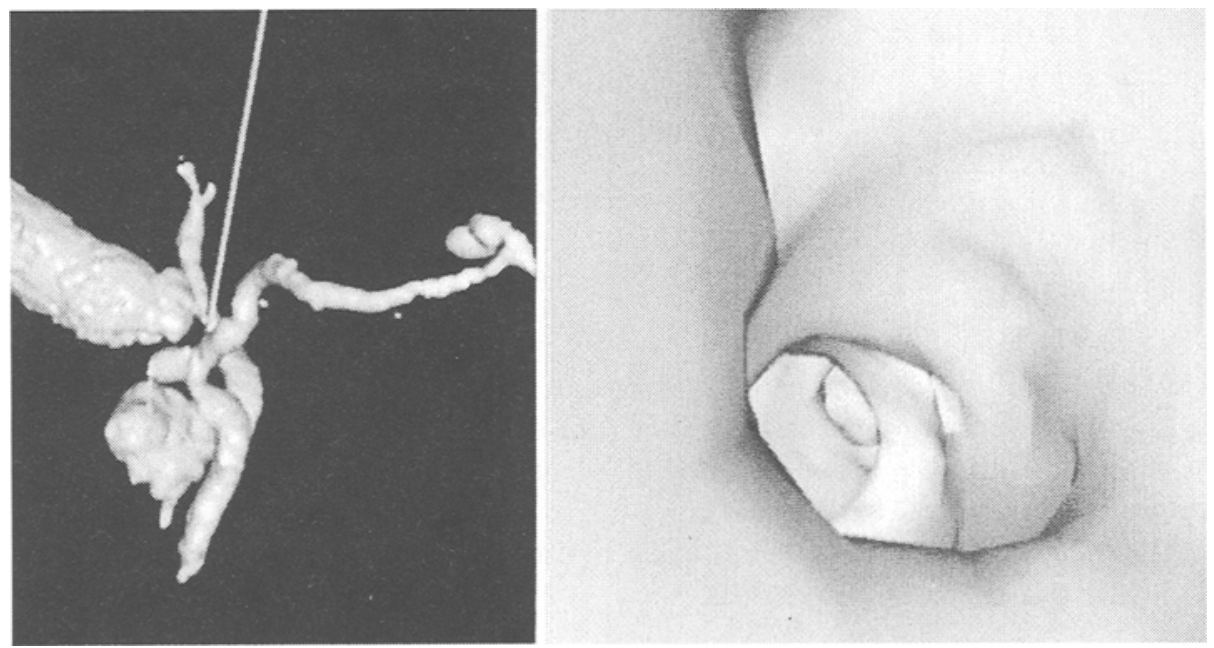

When treating low grade malignant tumors of the pancreas such as mucin-producing intraductal papillary tuomrs, minimally invasive surgical procedures have been advocated these days because of patient's quality of life6). For surgical planning of pancreatectomy, it is necessary to clarify the anatomical relationship among the tumor , the panceatic duct and the bile duct. Whether the bile duct can be preserved or not is one of the most important factor for choosing operative procedures. Preservation of the bile duct depends on the degree of the involvement by the tumors. When the bile duct is not involved by the tumor, we perform resection of the inferior head of the pancreas or duodenumpreserving pancreas head resection (DpPHR) according to the tumor extension to the Santorini"s pancreas. Anatomical identification of the lesion in the head of the pancreas is useful for surgical planning. 
Virtual endoscopy proved to have a diagnostic potential for pancreatic disease. The surfaces of the cystic lesions could be visualized in all cases of our serires. Real pancreatoscopy do not always demonstrate the view of inner space of the cystic lesions in the pancreas. All intraductal papillary adenocarcinoma $(n=4)$ had irregular surfaces illustrated by virtual endoscopy. 13 lesions of 14 benign lesions had smooth or slightly irregular surface. Preoperative diagnosis of MPPT is difficult, especially concerning the oncological feature of these kind of tumors. So virtual endoscopy would be useful to evaluate the oncological characters before the operation. The advantages of virtual endoscopy are summarized in Table 2.

Table2: Advantages of virtual endoscopy

1. visualization of inner surface of cystic lesions and the pancreatic duct

2. display of anatomical relationship among the lesion, the pancreatic duct and the bile duct

3. preoperative evaluation of oncological feature by observing the surface of cystic lesions

\section{Conclusion}

Virtual endoscopy of the pancreas proved to be a valuable method for clarifying the anatomical relationship between the pancreatic ducts and cystic lesions. Virtual endoscopy had a diagnostic potential to evaluate oncological features by observing the inner surface of the cystic lesions.

It is concluded that virtual endoscopy is useful for surgical planning of minimally invasive resection of the pancreas.

Acknowledgement: This study was supported by Japan association for the advancement of medical equipments. 


\section{References}

1)Jolesz FA, Lorensen WE, Kikinis R et al. Interactive Virtual Endoscopy. AJR Vol 169: 1229-1235, 1997

2)Jolesz FA, Lorensen WE, Kikinis R, et al. Virtual endoscopy: three-dimensional rendering of cross-sectional images for endoluminal visualization (abst). Radiology $469,193,1994$

3)Lorensen WE, Jolesz FA, Kikinis R. The exploration of cross-sectional data with a virtual endoscope. In: Satava RM, Morgan K, Sieburg HB et al. Interactive technology and the new pardigm for health-care: medicine meets virtual reality III proceedings. Amsterdam, Holland: IOS Press; 221-230. 1995

4)Ron Kikinis ,P. Langham Gleason, Thomas M. Moriarty et al. Computer Assisted Interactive Three-Dimensional Planning for Neurosurgical Procedures. Neurosurgery, Vol 38: 640-651,1996

5)Shin Nakajima, Hideki Atsumi, Abhir H. Bhalerao, et al. Computer-assisted Surgical Planning for Cerebrovascular Neurosurgery.Neurosurgery.Vol41,403-409,1997

6)Nakagohri T, Asano T, Takayama $W$ et al. Resecton of the Inferior Head of the Pancreas:Report of a case. Surgery Today. Vol 26,640-644,1996 